\title{
Lifestyle advice to cancer survivors: a qualitative study on the perspectives of health professionals
}

Adoption of positive health behaviours has shown promising effectiveness in reducing the high morbidity burden of cancer survivors $^{(1,2)}$. Health professionals (HPs) are well suited to provide healthy lifestyle advice but few survivors report receiving guidance from them ${ }^{(3,4)}$. We aimed to explore in depth HPs' perspective of lifestyle advice (heathy eating, physical activity, smoking, and alcohol) for cancer survivors.

Twenty-one HPs across the UK were interviewed using a semi-structured protocol. Two researchers analysed the data using the inductive six-phase thematic approach ${ }^{(5)}$. Inter-rater reliability was high (mean kappa $\left.=0.92\right)$. Participants $($ mean age $($ SD): 47.8 (8.0) years) were working in secondary care with breast, prostate, or colorectal cancer survivors. Nurses and physicians represented $43 \%$ and $33 \%$ of the sample, respectively.

Two main data-driven themes were identified: (1) factors influencing provision of lifestyle advice, and (2) optimal delivery of lifestyle advice to cancer survivors. Results suggested that perceived physical ability of survivors to follow lifestyle guidelines, potential for being perceived as blaming the survivors, and practical and cultural factors influenced provision of lifestyle advice. HPs tended to provide generic advice broadly based on lifestyle guidelines for the general population but tried to tailor it to the individual patient. Further factors included clinicians' knowledge of healthy lifestyle guidelines, feeling that they were not the 'right person' to provide advice, and lack of time and resources. HPs stressed that the optimal delivery of lifestyle advice should (a) be tailored to the individual and delivered throughout the cancer journey, (b) be focused on small and achievable changes framed as part of their treatment regimen, and (c) be cost-effective for wide-scale implementation.

In conclusion, incorporation of the identified factors influencing provision of lifestyle advice when developing HP training programmes and lifestyle interventions for cancer survivors could increase the probability of successful behaviour change and, thus, improved outcomes for cancer survivors.

1. Stacey FG, James EL, Chapman K, et al. (2015) J Cancer Surviv 9, 305-338.

2. Stolley M, Sheean P, Gerber B, et al. (2017) J Clin Oncol JCO2016719856.

3. Beeken RJ, Williams K, Wardle J, et al. (2016) Eur J Cancer Care (Engl) 25, 774-783.

4. Smith L, Croker H, Fisher A, et al. (2017) Eur J Cancer Care (Engl) $\mathbf{2 6 .}$

5. Braun V, Clarke V (2006) Qualit Res Psychol 3, 77-101. 